\title{
Análise envoltória de dados (DEA) como uma ferramenta para comparação de eficiência entre rotas de transporte de carga
}

\author{
Data envelopment analysis (DEA) as a tool for \\ benchmarking freight transport route efficiency
}

\section{Análisis de Envoltura de Datos (DEA) como uma herramienta para la comparación de eficiencia entre rutas de transporte de carga}

\author{
Isotilia Costa Melo \\ Mestre em Engenharia de Produção. \\ Escola de Engenharia de São Carlos, EESC, Brasil \\ Paulo Nocera Alves Junior \\ Doutor em engenharia de Produção \\ Escola de Engenharia de São Carlos, EESC, Brasil \\ Daisy Aparecida do Nascimento Rebelatto \\ Professora Associada \\ Escola de Engenharia de São Carlos, EESC, Brasil.
}

\begin{abstract}
Resumo O objetivo deste trabalho é apresentar a análise envoltória de dados (DEA) como uma possível ferramenta para comparar a eficiência de rotas de transporte de carga. Os Estados Unidos e o Brasil produzem mais de $80 \%$ dos grãos de soja do mundo. Isso representa 1,7\% das exportações norte-americanas, e 10\% das exportações brasileiras, que é uma economia menor. Transportar a soja, entre outros produtos de exportação, das fazendas até os principais portos, de forma mais eficiente, é o foco dos programas de investimento brasileiros. Uma ferramenta consolidada para a comparação de eficiência entre rotas irá ajudar os tomadores de decisão a direcionar investimentos. Este artigo determina as rotas das principais microrregiões produtoras até os principais portos de ambos os países, considerando a colheita de 2014. O uso de DEA SBM (Slack-Based Measure) e as variáveis iniciais são propostos. As variáveis são validadas por meio de correlação, regressão linear e análise de componentes principais (PCA). Após a aplicação do modelo, foi usado o método de desempate quantitativo do índice composto. Os resultados demonstraram que DEA pode ser usada para essa aplicação. Palavras-chaves: Análise Envoltória de Dados. Modelagem de Transporte. Soja. Slack-Based Measure. Eficiência de Rota.
\end{abstract}


Aвstract The purpose of this paper is to present data envelopment analysis (DEA) as a possible solution tool for benchmarking freight transport route efficiency. United States and Brazil produces more than $80 \%$ of the world soybeans. That represents $1.7 \%$ of American exports, but $10 \%$ of Brazilian exports, which is a smaller economy. Transporting soybeans, among other export products, from farmers to export ports more efficiently is the focus of Brazilian investment programs. A developed tool for benchmarking route will help decision makers to direct investments properly. This paper determines routes from main producing micro-regions to main export ports in both countries, considering the harvest of 2014. The use DEA SBM (Slack Based Measure) and initial variables are proposed. The variables are validated through correlation, linear regression and principal component analysis (PCA). After the application of the model, the quantitative tiebreaking method of composed index was applied. The results demonstrated that DEA can be used for this application.

Keywords: Data Envelopment Analysis. Freight Transport Modeling. Soybeans. Slack Based Measure. BraziL.

REsumen El objetivo de este trabajo es presentar un análisis de envoltura de datos (DEA) como una posible herramienta para comparar la eficiencia de rutas de transporte de carga. Los Estados Unidos y el Brasil producen más de $80 \%$ de los granos de soya en el mundo. Esto representa 1,7\% de las exportaciones norte-ameriacanas, y 10\% de las exportaciones brasileras, que es una economia menor. Transportar la soya entre otros productos de exportacíon de las haciendas hasta los principales puertos, de la forma más eficiente, es el foco de los programas de investimientos brasileros. Una herramienta consolidada para la comparacíon de eficiencia entre rutas ayudaria a los tomadores de decisión a direccionar inversiones. Este artículo determina las rutas de las principales micro-regiones productoras hasta los principales portos en ambos países, considerando la cosecha de 2014. El uso de DEA SBM (Slack-Based Measure) y las variables iniciales son propuestas. Las variables son validadas por medio de correlacíon, regresion linear y análisis de componentes principales (PCA). Despues de la aplicacíon de modelo, fue usado el método de desempate cuantitativo del índice compuesto. Los resultados demuestran que DEA puede ser usado para esta aplicacíon.

Palabras-claves: Análisis de Envoltura de Datos. Modelaje de Transporte. Soya. SlackBased Measure. Eficiencia de Ruta.

\section{INTRODUÇÃO}

A soja é considerada o $44^{\circ}$. produto mais importante da economia mundial, numa lista de 1.238 itens, elaborada pelo projeto The Observatory of Economic Complexity (OEC) do Laboratório de Mídia do Massachussets Institute of Technology (MIT) (SIMOES; HIDALGO, 2011). Em 2014, os Estados Unidos (EUA) produziram 41\% da soja do mundo e o Brasil, $40 \%$. Isso representou $1,7 \%$ do total de exportações norte-americano e $10 \%$ do brasileiro (SIMOES; HIDALGO, 2011). Os dois países são os maiores produtores mundiais de soja, 
somando $81 \%$ da produção mundial e, de acordo com a mesma fonte, têm a China como maior consumidor de seu produto. Além disso, ambos têm suas maiores regiões produtoras localizadas no centro territorial, tornando necessário o transporte da carga por centenas de quilômetros até os portos exportadores (SALIN, 2016).

A infraestrutura norte-americana para o transporte de soja dos agricultores até os principais portos de exportação está consolidada e tende a ser de baixo custo. Já o caso brasileiro é mais desafiador, o transporte de soja ainda enfrenta deficiências e se mostra mais oneroso. Para modificar a matriz de transporte, em 2007, o governo brasileiro criou o Plano de Aceleração do Crescimento 1 (PAC 1) e o integrou ao Plano Nacional de Logística e Transporte (PNLT). O PNLT é executado pelos Ministérios dos Transportes e da Defesa. Planeja-se executá-lo em três fases, entre 2008 e 2023 (SALIN, 2016). Outros países também possuem grande extensão territorial (como a Rússia, por exemplo) ou estão inseridos dentro de um contexto econômico semelhante ao do Brasil (como a China). Porém, o escoamento nesses países não apresenta características tão semelhantes entre si quanto o brasileiro e o norte-americano. Por exemplo, a Rússia, assim como a China, tem um vasto deserto central, rodeado por uma cordilheira, o que força as regiões agrícolas produtoras a estarem próximas dos centros exportadores (PALLOT; NEFEDOVA, 2003; BLOOMBERG NEWS, 2017). Dado o contexto exposto, uma comparação entre Brasil e EUA se mostra pertinente para direcionar as políticas de investimentos brasileiras rumo à consolidação já atingida pelos EUA.

A técnica análise envoltória de dados (DEA) é uma ferramenta não paramétrica para mensuração da eficiência de determinado grupo analisado. Cada unidade analisada é chamada de DMU (Decision Making Units). Na DEA, são inseridas as variáveis (de entrada ou de saída) características de cada DMU e a DEA retorna um ranking de eficiência. Existem diversos modelos DEA, que podem ter escala constante ou variável e orientação para entrada (inputs), para saída (outputs) ou ambas simultaneamente. A DEA foi inicialmente utilizada para mensuração de investimentos públicos (como saúde e educação), mas foi aplicada a diversos setores nas últimas décadas (ALMEIDA; MARIANO; REBELATTO, 2006). Emrouznejad e Yang (2017), ao reverem a aplicação da DEA entre 1978 e 2017, apontam transportes como estado da arte.

O objetivo do trabalho é explorar a análise envoltória de dados (DEA) como uma ferramenta para comparação de eficiência entre rotas de transporte de carga em diferentes contextos econômicos. A discussão limites de homogeneidade para aplicação da DEA foge do escopo deste trabalho, os leitores interessados podem consultar Melo (2017). Para alcançar esse objetivo, é necessário mostrar que os obstáculos encontrados por Panakagos (2016) podem ser superados com DEA.

Este artigo está estruturado em cinco subtópicos. O Subtópico 2 apresenta os conceitos fundamentais para o entendimento do artigo, i.e., o que são rotas e como elas são comparadas e a definição do modelo DEA-SBM (Slack-Based Measure). O subtópico 3 detalha o método, o 4 exibe os resultados seguidos de uma breve discussão. Por último, as considerações finais são debatidas no subtópico 5 . 


\section{CONCEITOS FUNDAMENTAIS}

Considerando a definição de Arnold (2006), corredores de transporte de carga incluem uma ou mais rotas que ligam centros de atividade econômica. Panagakos (2016) descreve o processo de desenvolvimento de um método para comparação de eficiência entre corredores de transporte de carga da União Europeia. Inicialmente, segundo o autor, pensou-se em 11 etapas. Os primeiros passos incluíam a escolha de um corredor com dados disponíveis e a determinação de seus segmentos críticos. Em seguida, a escolha de quatro ou cinco cargas típicas desse corredor. Depois, a partir da seleção de cadeias desse tipo de carga, se construiriam indicadores chave de desempenho (KPI) para as cadeias, se identificariam os obstáculos para cada KPI e se transformariam os KPI's de cadeias em KPI's de rotas. Finalmente, no passo 10, se transformaria o conjunto de valores de KPI's em um ranking de eficiência de rotas, para se poder compará-las quanto a sua eficiência em relação às demais. Pesos relativos devem ser dados a cada KPI no ranking. Esperava-se que diferentes participantes da cadeia propusessem diferentes pesos para esse cálculo, conforme seus interesses, que normalmente são antagônicos.

Panagakos (2016) aplicou esse método a um dos corredores economicamente mais importantes da Europa (Corredor Brenner). Entre os obstáculos enfrentados, foi mencionado que a amostra era muito pequena, o cenário resultante teve valor estatístico limitado. É importante lembrar que a DEA, proposta pelo presente trabalho, é não-paramétrica, portanto, não é afetada pelo tamanho da amostra. Depois de várias discussões, Panagakos (2016) decidiu pela exclusão da etapa 10 do método. O motivo para essa decisão foi a dificuldade de atribuir pesos aos KPI's. A DEA gera como resultado um ranking de unidades analisadas (chamadas DMU's, Decision Making Units). Para o ranking, os modelos DEA escolhem automaticamente os pesos que são mais favoráveis para cada DMU e ignoram pesos menos favoráveis, atribuindolhes zero (COELLI; RAO; BATTESE, 1998), resolvendo obstáculos encontrados por Panagakos (2016). A ideia de combinar DEA com análise de eficiência de rotas de transporte de carga também foi desenvolvida, simultânea e independemente do presente trabalho, por Oliveira e Cicolin (2016).

Em prol do maior entendimento do leitor a respeito de como funciona o modelo DEA ancorado em folgas (SBM), segue uma demonstração resumida, fundamentada no artigo de Tone (2001). Para maiores esclarecimentos, consulte o artigo original. Lidando com $n$ DMU's com matrizes, respectivamente, de inputs e outputs, $X=\left(x_{i j}\right) \in \mathbb{R}^{m x n}$ e $Y=\left(y_{i j}\right) \in \mathbb{R}^{s x n}$. Assume-se que os dados são positivos, i.e., $X>0$ e $Y>0$. Conforme a Equação 1, a função produção é definida como: $P=\{(x, y) \mid x \geq X \lambda, y \leq Y \lambda, \lambda \geq 0\}$

Onde $\lambda$ é um vetor não negativo em $\mathbb{R}^{n}$. É possível impor algumas restrições em $\lambda$, como $\sum_{j=1}^{n} \lambda_{j}=1$ (como no modelo BBC), se for necessário modificar o conjunto de produção. Considerando uma expressão para descrever certa DMU $\left(x_{0}, y_{0}\right)$, conforme Equações 2 e 3 : 


$$
\begin{aligned}
& x_{0}=X \lambda+s^{-}, \\
& y_{0}=Y \lambda+s^{+},
\end{aligned}
$$

$\operatorname{Com} \lambda \geq 0, s^{-} \geq 0$ e $s^{+} \geq 0$. O vetor $s^{-} \in \mathbb{R}^{m}$ e $s^{+} \in \mathbb{R}^{s}$ indica, respectivamente, o excesso de inputs e a falta de outputs dessa expressão, isto é, as folgas (SBM, em inglês, significa mensuração ancorada em folgas). A partir da condição $\lambda \geq 0$ e $X>0$, chega-se à Equação 4: $x_{0} \geq s^{-}$.

Usando $s^{-}$e $s^{+}$, pode-se definir o índice Q, para eficiência, de acordo com a Equação 5:

$$
\rho=\frac{1-(1 / m) \sum_{i=1}^{m} s_{i}^{-} / x_{i 0}}{1-(1 / s) \sum_{r=1}^{s} s_{r}^{+} / y_{r 0}}
$$

Pode-se observar que $\varrho$ atende a duas das quatro propriedades mais importantes para realizar mensurações de eficiência: 1) as medições devem ser invariantes com relação às unidades de medida; 2) as medições devem decrescer de maneira monótona para toda folga de inputs e outputs. A partir da quarta propriedade (a medição deve ser determinada apenas consultando o conjunto referência de onde a DMU é originária), resulta na Equação 6:

$$
0<\rho \leq 1 \text {. (6) }
$$

No esforço de estimar a eficiência de $\left(x_{0}, y_{0}\right)$, Tone (2001) formula programa fracionário em $\lambda, s^{-}$e $s^{+}$conforme Equações 7, 8, 9 e 10.

$$
\begin{aligned}
& \text { (SBM) } \\
& \text { Minimizar } \rho=\frac{1-(1 / m) \sum_{i=1}^{m} s_{i}^{-} / x_{i 0}}{1-(1 / s) \sum_{r=1}^{s} s_{r}^{+} / y_{r 0}}
\end{aligned}
$$

Para linearizar, Tone (2001) multiplica uma variável escalar positiva $t$ para tanto o numerador quanto o denominador de $\varrho$. Isso não causa alterações em $\varrho$. Ajusta-se $t$ para que o denominador se torne 1. Assim, esse termo é movido para as restrições. O objetivo passa ser minimizar o numerador, resultando na reformulação das Equações 11, 12, 13, 14 e 15:

$$
\text { Minimizar } \tau=t-(1 / m) \sum_{i=1}^{m} s_{i}^{-} / x_{i 0}
$$




$$
\begin{aligned}
& \text { Sujeito a } 1=t+(1 / s) \sum_{r=1}^{s} s_{r}^{+} / y_{r 0}, \\
& x_{0}=X \lambda+s^{-} \\
& y_{0}=Y \lambda+s^{+} \\
& \lambda \geq 0 s^{-} \geq 0, s^{+} \geq 0 .
\end{aligned}
$$

O problema já citado é não linear uma vez que contém termos não lineares $t s_{r}^{+}(r=1, \ldots, s)$. No entanto, pode-se transformá-lo num problema linear definido pela Equação 16:

$$
S^{-}=t s^{-}, S^{+}=t s^{+}{ }_{\mathrm{e}} \Lambda=t \lambda .
$$

Assim, a programação linear se expressa pelas Equações 17, 18, 19, 20 e 21:

$$
\begin{aligned}
& \text { Minimizar } \tau=t-(1 / m) \sum_{i=1}^{m} \boldsymbol{S}_{i}^{-} / x_{i 0}, \\
& \text { Sujeito a } 1=t+(1 / s) \sum_{r=1}^{s} \boldsymbol{S}_{r}^{+} / y_{r 0}, \\
& \boldsymbol{t} x_{0}=X \boldsymbol{\Lambda}+\boldsymbol{S}^{-} \\
& \boldsymbol{t} y_{0}=Y \boldsymbol{\Lambda}+\boldsymbol{S}^{+} \\
& \boldsymbol{\Lambda} \geq 0, \boldsymbol{S}^{-} \geq 0, \boldsymbol{S}^{+} \geq 0 \mathrm{e}^{t>0}
\end{aligned}
$$

A solução ótima de $\left(\tau^{*}, \mathrm{t}^{*}, \Lambda^{*}, \mathrm{~S}^{-*}, \mathrm{~S}^{+*}\right)$ é dada pela Equação 22:

$$
\rho^{*}=\tau^{*}, \lambda^{*}=\Lambda^{*} / t^{*}, s^{-*}=S^{-*} / t^{*}, s^{+}=S^{+*} / t^{+}(22)
$$

Ancorado nessa solução, define-se uma DMU $\left(\mathrm{x}_{0}, \mathrm{y}_{0}\right)$ como eficiente, no modelo SBM, quando $\tilde{\mathrm{n}}^{*}=1$.

\section{Métodos}

O tipo de carga, soja, foi predefinido em prol do desenvolvimento econômico nacional. A Figura 1 explica o método empregado para o desenvolvimento do ranking de eficiência de transporte, usando DEA.

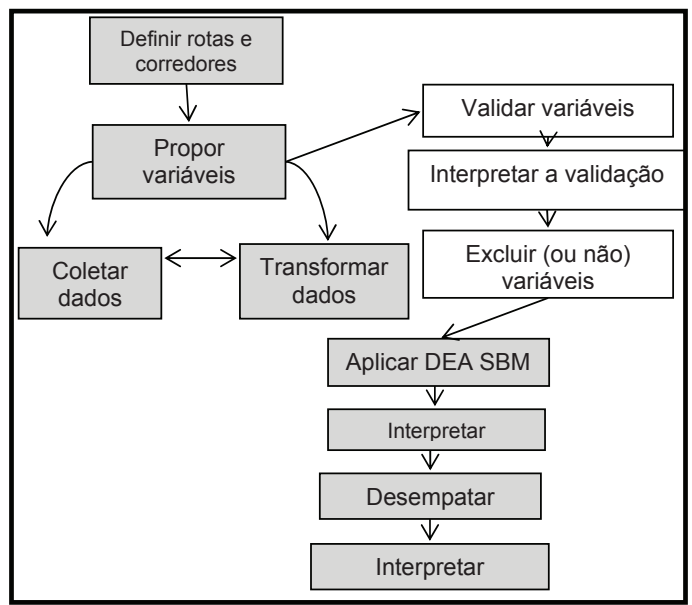

Figura 1 - Método proposto para construção do ranking de eficiência em rotas de transporte de carga. 
Em todo o texto, os conceitos de rota e corredor seguem as definições de Panagakos (2016): uma rota é uma alternativa de transporte entre uma origem e um destino, e corredor é um conjunto de rotas que ligam dois pontos de relevância econômica. No presente estudo, corredores ligam microrregiões produtoras a portos exportadores. As rotas foram definidas considerando a colheita de 2014 (um ano não afetado por eventos climáticos extraordinários). As rotas foram estabelecidas a partir das três principais microrregiões produtoras de cada Estado até os principais portos de exportação. No Brasil, de Rio Grande do Sul (RS), Paraná (PR), Mato Grosso do Sul (MS), Goiás (GO), Mato Grosso (MT) até os portos de Santos, Paranaguá e Rio Grande. Nos EUA, de Iowa (IA), Illinois (ILL), Missouri (MO), Minnesota (MN), e Indiana (IN) até os portos do Golfo do Mississipi e Pacific Northwest Complex (NPW) (SALIN, 2015). As distâncias rodoviárias, para ambos os países, foram determinadas usando o Google Earth (2016).

Para determinar a quantidade de soja colhida, no Brasil, foram consultados Imea (2015), Seab (2015), Emater (2014), Siga (2015) e Goiás (2015) e, para determinar rotas e corredores, foram consultados Ibge (2015), Antaq (2011), Ahrana (2005, 2012, 2013), Macrologística (2011, 2013), ALL (2016), Ferroeste (2016), Aliança (2016), Vieira (2002), Ojima (2004), Rocha e Parre (2009). Foram identificados nove corredores e 72 rotas.

Para determinar a quantidade de soja colhida, nos EUA, foi consultado Nass (2016), e para determinação de rotas e corredores, foram consultados Casavant et al. (2011), Salin (2015), US Corps of Engineers (2016), do Waterways Council (2016) e National Oceanic and Atmospheric Administration (NOAA, 2016). Foram identificados cinco corredores e 30 rotas.

Todas as rotas receberam um código, de acordo com os modais de transporte existentes e o país. Rotas norte-americanas começam com E. Se houver modal rodoviário, o código da rota recebe $R$. Se houver modal hidroviário fluvial, recebe $H$. Se houver modal ferroviário, recebe $F$.

Golany e Roll (1989), ao discutirem os critérios para escolha de um modelo DEA adequado e das variáveis, apontam a necessidade de ter em mente o contexto integral do problema. Um dos meios apontados para isso é a revisão de literatura. A proposta inicial de variáveis foi: combustível consumido (diesel), como input, colheita (assumindo toda a colheita foi transportada), como output, emissões e fator de descarga, como outputs indesejados. As duas primeiras variáveis são utilizadas por Oliveira e Cicolin (2016) numa aplicação similar de DEA. Emissões é explicitamente citada por Panagakos (2016). Esse autor enfoca que empregou outras variáveis ambientais, mas não enumera todas.

A variável diesel foi pensada para representar o custo de transporte. Pois o diesel é o combustível presente em todos os modais de ambos os países, cujo preço é regido por cotações internacionais. Ela foi calculada usando o método desenvolvido por Texas Instruments (TTI, 2012). A variável emissões é encontrada com frequência na literatura (CAMIOTO; MARIANO; REBELATTO, 2014), para seu cálculo foi adotado o método bottom-up com os fatores do Greenhouse Gas Protocol (2016). A variável fator de descarte foi pensada para representar os resíduos gerados pelos modais, após um ciclo econômico. Ela é calculada por meio da multiplicação de duas frações. A primeira fração é a duração de um ciclo econômico (assumido 100 anos) pela idade média da frota daquele modal. A segunda fração é o tamanho da frota de um modal necessária para transportar a colheita da microrregião estudada dividida pela frota necessária para transportar toda colheita nacional. No caso de uma rota com vários modais, o fator de descarte total é a soma do fator de descarte para cada modal. O Quadro 1 mostra os dados utilizados. 


\begin{tabular}{|c|c|c|c|c|c|c|c|c|c|}
\hline Cód. & Diesel (1) & $\begin{array}{l}\text { Emissões } \\
(\mathrm{kg} \text { de } \\
\left.\mathrm{CO}_{2 \mathrm{eq}}\right)\end{array}$ & $\begin{array}{l}\text { Fator de } \\
\text { Descarte }\end{array}$ & $\begin{array}{c}\text { Produção } \\
\text { (t) }\end{array}$ & Cód. & Diesel (1) & $\begin{array}{l}\text { Emissões } \\
(\mathrm{kg} \text { de } \\
\left.\mathrm{CO}_{2 \mathrm{eq}}\right)\end{array}$ & $\begin{array}{l}\text { Fator de } \\
\text { Descarte }\end{array}$ & Produção (t) \\
\hline $\mathbf{R} 1$ & 621988,69 & 71410,53 & 2,02 & 9020409,31 & RF12 & 774569,96 & 52352,94 & 1,17 & 4141338,00 \\
\hline $\mathbf{R} 2$ & 100228,12 & 11507,19 & 0,53 & 2348669,66 & FH1 & 117147,41 & 1019,56 & 0,28 & 2348669,66 \\
\hline $\mathbf{R} 3$ & 35791,44 & 4109,22 & 0,27 & 1197689,90 & RFH1 & 473538,79 & 8901,80 & 3,08 & 9020409,31 \\
\hline R4 & 255876,86 & 29377,23 & 1,02 & 4527862,00 & RHR1 & 752027,00 & 41477,19 & 2,90 & 5732615,00 \\
\hline R5 & 333271,71 & 38262,93 & 1,02 & 4527862,00 & RHR2 & 713898,44 & 38398,32 & 2,90 & 5732615,00 \\
\hline R6 & 181253,64 & 20809,74 & 0,99 & 4415811,00 & RHR3 & 174680,08 & 9436,63 & 0,69 & 1356822,00 \\
\hline $\mathbf{R} 7$ & 358399,55 & 41147,86 & 0,99 & 4415811,00 & RHR4 & 165655,64 & 8707,91 & 0,69 & 1356822,00 \\
\hline R8 & 243263,69 & 27929,11 & 0,79 & 3504301,00 & RHR5 & 220505,70 & 15262,83 & 0,65 & 1284631,00 \\
\hline R9 & 414811,45 & 47624,51 & 0,79 & 3504301,00 & RHR6 & 211961,42 & 14572,88 & 0,65 & 1284631,00 \\
\hline R10 & 418526,12 & 48050,99 & 0,84 & 3737616,41 & RHR7 & 81251,73 & 6762,58 & 0,17 & 327876,67 \\
\hline R11 & 463725,20 & 53240,30 & 0,84 & 3737616,41 & RHR8 & 79070,98 & 6586,48 & 0,17 & 327876,67 \\
\hline $\mathbf{R} 12$ & 163806,18 & 18806,59 & 0,28 & 1220739,25 & RHR9 & 39383,05 & 2986,05 & 0,10 & 196210,00 \\
\hline R13 & 162812,55 & 18692,51 & 0,28 & 1220739,25 & RHR10 & 38078,02 & 2880,67 & 0,10 & 196210,00 \\
\hline R14 & 72966,64 & 8377,30 & 0,12 & 526437,12 & RHR11 & 26440,07 & 1955,26 & 0,07 & 138044,60 \\
\hline R15 & 59560,86 & 6838,19 & 0,12 & 526437,12 & RHR12 & 25521,92 & 1881,12 & 0,07 & 138044,60 \\
\hline R16 & 851893,26 & 97805,87 & 1,29 & 5732615,00 & RHF1 & 689834,35 & 18797,54 & 1,96 & 5732615,00 \\
\hline $\mathbf{R} 17$ & 715243,71 & 82117,14 & 1,29 & 5732615,00 & RHF2 & 159960,04 & 4068,71 & 0,47 & 1356822,00 \\
\hline R18 & 199421,28 & 22895,56 & 0,31 & 1356822,00 & RHF3 & 203999,59 & 10176,56 & 0,44 & 1284631,00 \\
\hline R19 & 163449,72 & 18765,67 & 0,31 & 1356822,00 & RHF4 & 77694,63 & 5465,41 & 0,12 & 327876,67 \\
\hline $\mathbf{R} 20$ & 144894,43 & 16635,33 & 0,29 & 1284631,00 & RHF5 & 37254,38 & 2209,79 & 0,07 & 196210,00 \\
\hline $\mathbf{R} 21$ & 2138823,38 & 245558,32 & 2,20 & 9836300,00 & RHF6 & 24666,35 & 1408,70 & 0,05 & 138044,60 \\
\hline R22 & 1906640,95 & 218901,45 & 2,20 & 9836300,00 & EH1 & 33987,63 & 124,67 & 0,13 & 1369420,86 \\
\hline $\mathbf{R} 23$ & 1167677,66 & 134061,08 & 1,32 & 5886300,00 & ERH1 & 119700,58 & 3726,77 & 0,42 & 2393696,94 \\
\hline $\mathbf{R} 24$ & 1060902,91 & 121802,27 & 1,32 & 5886300,00 & ERH2 & 116068,79 & 3710,10 & 0,40 & 2285570,44 \\
\hline $\mathbf{R} 25$ & 1090712,86 & 125224,75 & 0,93 & 4141338,00 & ERH3 & 98935,56 & 2794,63 & 0,37 & 2083632,71 \\
\hline R26 & 921688,49 & 105819,06 & 0,93 & 4141338,00 & ERH4 & 119812,45 & 2422,38 & 0,38 & 2171837,58 \\
\hline
\end{tabular}




\begin{tabular}{|c|c|c|c|c|c|c|c|c|c|}
\hline F1 & 96249,41 & 1767,14 & 0,14 & 2348669,66 & ERH5 & 156640,84 & 5654,04 & 0,39 & 2213830,83 \\
\hline F2 & 158919,08 & 2917,76 & 0,27 & 4527862,00 & ERH6 & 111765,68 & 2592,89 & 0,35 & 1992434,14 \\
\hline F3 & 119688,69 & 2197,49 & 0,27 & 4415811,00 & ERH7 & 54516,37 & 2112,17 & 0,18 & 1003565,21 \\
\hline F4 & 151921,76 & 2789,29 & 0,21 & 3504301,00 & ERH8 & 82348,96 & 2807,86 & 0,35 & 1948290,88 \\
\hline F5 & 40735,23 & 747,90 & 0,04 & 526437,12 & ERH9 & 37480,90 & 838,76 & 0,20 & 1089157,42 \\
\hline RH1 & 672621,23 & 47964,68 & 2,55 & 9020409,31 & ERH10 & 119322,24 & 3214,23 & 0,32 & 1784318,53 \\
\hline RH2 & 146729,79 & 9227,81 & 0,67 & 2348669,66 & ERH11 & 109157,14 & 2649,59 & 0,30 & 1706645,99 \\
\hline RH3 & 86800,88 & 6080,74 & 0,34 & 1197689,90 & ERH12 & 81459,51 & 943,45 & 0,27 & 1537856,53 \\
\hline RH4 & 529047,97 & 55584,85 & 2,55 & 9020409,31 & ERH13 & 96634,33 & 4310,89 & 0,26 & 1452890,27 \\
\hline RH5 & 91868,74 & 9205,19 & 0,67 & 2348669,66 & ERH14 & 48944,99 & 1287,87 & 0,19 & 1065670,59 \\
\hline RH6 & 32503,49 & 3047,25 & 0,34 & 1197689,90 & ERF1 & 570521,24 & 11560,99 & 0,64 & 2393696,94 \\
\hline RH7 & 719267,99 & 32235,16 & 2,55 & 9020409,31 & ERF2 & 522894,27 & 9171,40 & 0,61 & 2285570,44 \\
\hline RH8 & 149589,90 & 4066,21 & 0,67 & 2348669,66 & ERF3 & 479331,92 & 8586,39 & 0,56 & 2083632,71 \\
\hline RH9 & 91880,29 & 3864,33 & 0,34 & 1197689,90 & ERF4 & 544326,78 & 9395,73 & 0,58 & 2171837,58 \\
\hline RF1 & 210252,40 & 8811,34 & 1,28 & 4527862,00 & ERF5 & 617219,30 & 14906,10 & 0,59 & 2213830,83 \\
\hline RF2 & 397403,95 & 11848,76 & 2,55 & 9020409,31 & ERF6 & 461537,86 & 5387,83 & 0,53 & 1992434,14 \\
\hline RF3 & 531723,81 & 33152,59 & 1,06 & 3737616,41 & ERF7 & 208698,23 & 2950,14 & 0,27 & 1003565,21 \\
\hline RF4 & 153793,30 & 8546,38 & 0,35 & 1220739,25 & ERF8 & 445556,94 & 9178,79 & 0,52 & 1948290,88 \\
\hline RF5 & 779261,84 & 65290,76 & 1,62 & 5732615,00 & ERF9 & 273995,17 & 7259,94 & 0,29 & 1089157,42 \\
\hline RF6 & 182861,62 & 15272,19 & 0,39 & 1356822,00 & ERF10 & 281827,76 & 3920,75 & 0,48 & 1784318,53 \\
\hline RF7 & 2031355,18 & 191737,03 & 2,78 & 9836300,00 & ERF11 & 266092,99 & 3453,89 & 0,46 & 1706645,99 \\
\hline RF8 & 1062983,12 & 97216,66 & 1,67 & 5886300,00 & ERF12 & 238382,38 & 2993,22 & 0,41 & 1537856,53 \\
\hline RF9 & 1028611,68 & 100629,55 & 1,17 & 4141338,00 & ERF12 & 286951,62 & 4462,56 & 0,39 & 1452890,27 \\
\hline RF10 & 1564074,88 & 92699,26 & 2,78 & 9836300,00 & ERF13 & 230205,75 & 4959,08 & 0,29 & 1065670,59 \\
\hline RF11 & 772399,06 & 36692,55 & 1,67 & 5886300,00 & ERF14 & 334596,85 & 5381,09 & 0,37 & 1369420,86 \\
\hline
\end{tabular}

Quadro 1 - Dados utilizados. 
Para validação das variáveis, foi feita correlação linear, regressão linear e análise dos componentes principais, com auxílio do software STATA. A correlação pode indicar se as variáveis estão corretamente classificadas como inputs e outputs (GOLANY; ROLL, 1989). A regressão linear determina a reta da equação e permite extrapolar os resultados de relação entre as variáveis dependentes e independentes (IBID.). Análise de Componentes Principais (PCA) é um método simples de análise multivariada. O objetivo da análise é tomar $p$ variáveis e encontrar suas combinações para produzir índices (chamados componentes principais), $\operatorname{Comp}_{1}, \operatorname{Comp}_{2}, \operatorname{Comp}_{3} . ., \operatorname{Comp}_{p}$, que não estão relacionados em ordem de importância e descrevendo a variação de dados. A falta de correlação significa que os índices são medição diferente das correlações de dados e a ordem é tal que, $\operatorname{Var}\left(\mathrm{Comp}_{1}\right) \geq \operatorname{Var}\left(\mathrm{Comp}_{2}\right) \geq \operatorname{Var}\left(\mathrm{Comp}_{3}\right) \geq \ldots \geq \operatorname{Var}\left(\mathrm{Comp}_{\mathrm{p}}\right)$

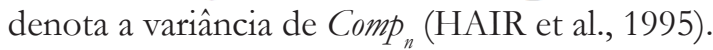

O modelo DEA SBM (Slack Based Measure) foi aplicado com auxílio do software MATLAB. Sendo, $X=\left(x_{i j}\right) \in R^{m x n}$ e $\mathrm{Y}=\left(\mathrm{y}_{\mathrm{ij}}\right) \in \mathrm{R}^{\text {sxn }}$, respectivamente, inputs e outputs, assumidos positivos, de uma DMU $\left(\mathbf{x}_{0} \cdot \mathrm{y}_{0}\right)$. Esse modelo SBM, criado por Tone (2001), é um avanço do modelo aditivo (MARIANO, SOBREIRO; REBELATTO; 2015), por ser invariante à unidade de medida, em que a eficiência obtida é um valor entre 0 e 1 . A eficiência desse modelo é calculada com base na média das folgas relativas, eliminando o problema dos falsos eficientes (DMUs eficientes, mas com folgas), e não necessita de orientação (minimiza inputs e maximiza outputs ao mesmo tempo).

Não se deseja maximizar as variáveis emissões e fator de descarte, apesar de elas serem outputs, pois elas representam um impacto negativo ao meio ambiente e à sociedade. Esse tipo de variável é chamado de output indesejável. Para minimizá-las com o aumento da eficiência, elas foram inseridas como inputs.

Um problema muitas vezes encontrado no ranking gerado por meio do DEA é o número de empates, optou-se pelo uso do método quantitativo de desempate do índice composto (LETA et al., 2005). Esse índice composto é a média normatizada da soma das eficiências padrão e invertida.

\section{RESULTADOS E DISCUSSÕES}

O Quadro 2 mostra a correlação entre as variáveis. Observa-se que emissões têm uma relação muito forte com diesel $(0,9274)$ e o fator de descarte com produção $(0,9233)$. As relações entre diesel e fator de descarte; diesel e produção; e produção e emissões são fortes (respectivamente, 0,7311, 0,7792 e 0,9233). A relação entre fator de descarte e emissões é moderada (0,5998). O fato de o imput ter uma relação, no mínimo, forte no mesmo sentido dos inputs sugere que o modelo possui validade. 
Conforme Golany e Roll (1989), o fato de os outputs possuírem relação, no mínimo, moderada entre si, pode indicar redundância de variáveis no modelo. Em desenvolvimentos posteriores da DEA, essa interpretação foi mantida e agregada a outras técnicas, como, por exemplo, PCA (ADLER; YAZHEMSKY, 2010; COOK; TONE; ZHU, 2014).

\begin{tabular}{lrrrr}
\hline & \multicolumn{1}{c}{ Diesel } & Emissões & \multicolumn{1}{c}{$\begin{array}{c}\text { Fator de } \\
\text { Descarte }\end{array}$} & Produção \\
\hline Diesel & 1 & & & \\
Emissões & 0,9274 & 1 & & \\
Fator de Descarte & 0,7311 & 0,5998 & 1 & 1 \\
Produção & 0,7792 & 0,7019 & 0,9233 & 1 \\
\hline
\end{tabular}

Quadro 2 - Matriz de correlação entre input e outputs.

O Quadro 3 mostra a regressão linear entre as variáveis dependentes, outputs, e a variável independente, imput. O Fator $\mathrm{R}$ indica o encaixe dessa relação (independente-dependentes) numa linha reta. Quanto mais alto $R$-sq, tanto mais perfeitamente as variáveis se encaixam e podem ser explicadas no modelo. E o Fator $P$ é comparável ao erro. Observa-se que existe uma alta relação para todas as variáveis e uma baixa relação com o erro.

\begin{tabular}{lcrrrrr}
\hline \multicolumn{1}{c}{ Equaçoes } & Obs & Parms & RMSE & R-sq & \multicolumn{1}{c}{$F$} & \multicolumn{1}{c}{ Fator $P$} \\
\hline F. Descarte & 102 & 2 & 0,5333174 & 0,5345 & 114,8026 & 0,0000 \\
Emissões & 102 & 2 & 17146,14 & 0,8600 & 614,4568 & 0,0000 \\
Produção & 102 & 2 & 1658213 & 0,6070 & 154,4762 & 0,0000 \\
\hline
\end{tabular}

Quadro 3 - Regressão linear das variáveis dependentes em relação à variável independente consumo de combustível (diesel).

A análise de PCA indicou que os dois primeiros componentes principais explicam 96,80\% do modelo. Como ilustrado no Quadro 4, as variáveis apresentaram uma proporcionalidade semelhante em ambos os componentes, não justificando a exclusão de qualquer delas. Dadas as considerações até aqui mencionadas, as variáveis foram consideradas validadas.

\begin{tabular}{lcc}
\hline$P C A$ & Comp1 & Comp2 \\
Variáveis & 0,5159 & 0,3757 \\
\hline Diesel & 0,4842 & 0,6048 \\
Emissões & 0,4882 & $-0,5755$ \\
F. Descarte & 0,5110 & $-0,4025$ \\
Produção & \\
\hline
\end{tabular}

Quadro 4 - Composição dos componentes principais. 
A execução do modelo DEA-SBM inicialmente resultou em 12 DMU's com 100\% de eficiência. Após aplicação do método de desempate do índice composto (LETA et al., 2005) não houve mais empates, a única DMU 100\% eficiente foi a EH1, que representa a rota norte-americana exclusivamente hidroviária partindo do distrito de New Madrid, Missouri, até o Golfo do Mississipi. Os resultados estão compilados no Quadro 5.

\begin{tabular}{|c|c|c|c|c|c|c|}
\hline Rank & Cód. & $\begin{array}{c}\text { Eficiência } \\
\text { Padrão }\end{array}$ & $\begin{array}{l}\text { Eficiência } \\
\text { Invertida }\end{array}$ & $\begin{array}{c}\text { Índice } \\
\text { Composto }\end{array}$ & $\begin{array}{l}\text { Distância } \\
\text { Total(km) }\end{array}$ & Rotas \\
\hline 1 & EH1 & 1,0000 & 1,0000 & 1,0000 & 650,3 & New Madrid - Golfo do Mississipi \\
\hline 2 & F3 & 1,0000 & 0,9692 & 0,9841 & 414,7 & Guarapuava - Paranaguá \\
\hline 3 & F2 & 1,0000 & 0,9683 & 0,9785 & 537,0 & Londrina - Paranaguá \\
\hline 4 & F1 & 1,0000 & 0,9571 & 0,9742 & 627,0 & Santa Maria - Rio Grande \\
\hline 5 & F5 & 1,0000 & 0,9531 & 0,9459 & 1183,9 & Chapadão do Sul - Santos \\
\hline 6 & RH6 & 1,0000 & 0,9484 & 0,9347 & 245,5 & Bagé - Rio Grande \\
\hline 7 & RF2 & 1,0000 & 0,9456 & 0,9333 & 639,0 & Ijuí - Rio Grande \\
\hline 8 & F4 & 0,9074 & 0,9434 & 0,9265 & 663,3 & Cascavel - Paranaguá \\
\hline 9 & FH1 & 0,7188 & 0,9166 & 0,8440 & 640,0 & Santa Maria - Rio Grande \\
\hline 10 & R1 & 1,0000 & 0,9105 & 0,7998 & 593,0 & Ijuí - Rio Grande \\
\hline 11 & ERH12 & 0,5259 & 0,9045 & 0,7395 & 1286,3 & Faribault - Golfo do Mississipi \\
\hline 12 & ERH9 & 0,5338 & 0,9038 & 0,7386 & 745,7 & Montgomery - Golfo do Mississipi \\
\hline 13 & RH7 & 0,7301 & 0,9011 & 0,7066 & 804,0 & Ijuí - Rio Grande \\
\hline 14 & ERH8 & 0,4823 & 0,8918 & 0,6917 & 796,0 & Clinton - Golfo do Mississipi \\
\hline 15 & RF1 & 0,5330 & 0,8914 & 0,6913 & 634,5 & Londrina - Paranaguá \\
\hline 16 & R3 & 0,5292 & 0,8872 & 0,6859 & 257,0 & Bagé - Rio Grande \\
\hline 17 & ERH3 & 0,4596 & 0,8850 & 0,6820 & 961,4 & McLean - Golfo do Mississipi \\
\hline 18 & RH4 & 0,7411 & 0,8695 & 0,6808 & 516,5 & Ijuí - Rio Grande \\
\hline 19 & ERH4 & 0,4447 & 0,8689 & 0,6806 & 1224,3 & Plymouth - Golfo do Mississipi \\
\hline 20 & ERH14 & 0,4472 & 0,8666 & 0,6788 & 951,5 & Audrain - Golfo do Mississipi \\
\hline 21 & ERH1 & 0,4381 & 0,8593 & 0,6647 & 977,6 & Edgard - Golfo do Mississipi \\
\hline 22 & ERH6 & 0,4227 & 0,8552 & 0,6633 & 1204,4 & Webster - Golfo do Mississipi \\
\hline 23 & RH1 & 0,7095 & 0,8498 & 0,6595 & 710,0 & Ijuí - Rio Grande \\
\hline 24 & ERH2 & 0,4304 & 0,8427 & 0,6588 & 982,6 & Champaign - Golfo do Mississipi \\
\hline 25 & RF10 & 1,0000 & 0,8362 & 0,6579 & 1981,0 & Canarana - Santos \\
\hline 26 & R6 & 0,5097 & 0,8277 & 0,6481 & 353,0 & Guarapuava - Paranaguá \\
\hline 27 & ERH11 & 0,3776 & 0,8274 & 0,6312 & 1356,6 & Cottonwood - Golfo do Mississipi \\
\hline 28 & ERH7 & 0,3828 & 0,8141 & 0,6190 & 961,9 & Knox - Golfo do Mississipi \\
\hline 29 & ERH10 & 0,3570 & 0,8088 & 0,6129 & 1375,3 & Lac Qui Parle - Golfo do Mississipi \\
\hline 30 & RHF6 & 1,0000 & 0,7939 & 0,6124 & 1886,0 & Primavera do Leste - Santos \\
\hline 31 & RH8 & 0,3649 & 0,7865 & 0,6121 & 666,0 & Santa Maria - Rio Grande \\
\hline
\end{tabular}




\begin{tabular}{|c|c|c|c|c|c|c|}
\hline 32 & RH5 & 0,4285 & 0,7810 & 0,6112 & 348,5 & Santa Maria - Rio Grande \\
\hline 33 & $\mathbf{R} 2$ & 0,4099 & 0,7788 & 0,5954 & 367,0 & Santa Maria - Rio Grande \\
\hline 34 & ERH5 & 0,3566 & 0,7683 & 0,5920 & 1298,2 & Woodbury - Golfo do Mississipi \\
\hline 35 & $\mathbf{R} 4$ & 0,4741 & 0,7536 & 0,5912 & 486,0 & Londrina - Paranaguá \\
\hline 36 & RHF1 & 0,5057 & 0,7504 & 0,5786 & 1389,0 & Jataí - Santos \\
\hline 37 & ERH13 & 0,3307 & 0,7500 & 0,5697 & 1083,2 & Nodaway - Golfo do Mississipi \\
\hline 38 & ERF12 & 0,2521 & 0,7284 & 0,5441 & 2986,1 & Faribault - NPW \\
\hline 39 & ERF11 & 0,2583 & 0,7110 & 0,5430 & 2991,9 & Cottonwood - NPW \\
\hline 40 & R22 & 1,0000 & 0,7084 & 0,5399 & 1667,0 & Canarana - Santos \\
\hline 41 & RH2 & 0,3267 & 0,6856 & 0,5385 & 606,0 & Santa Maria - Rio Grande \\
\hline 42 & ERF10 & 0,2574 & 0,6832 & 0,5357 & 3004,9 & Lac Qui Parle - NPW \\
\hline 43 & R5 & 0,4406 & 0,6756 & 0,5347 & 633,0 & Londrina - Santos \\
\hline 44 & RH9 & 0,2670 & 0,6691 & 0,5229 & 778,0 & Bagé - Rio Grande \\
\hline 45 & RF11 & 0,5029 & 0,6564 & 0,5185 & 1742,0 & Primavera do Leste - Santos \\
\hline 46 & R8 & 0,3795 & 0,6529 & 0,5179 & 597,0 & Cascavel - Paranaguá \\
\hline 47 & ERF6 & 0,2456 & 0,6514 & 0,5069 & 4498,5 & Webster - NPW \\
\hline 48 & R7 & 0,4151 & 0,6435 & 0,5068 & 698,0 & Guarapuava - Santos \\
\hline 49 & RFH1 & 1,0000 & 0,6365 & 0,5000 & 645,0 & Ijuí - Rio Grande \\
\hline 50 & RH3 & 0,2644 & 0,6288 & 0,4877 & 692,0 & Bagé - Rio Grande \\
\hline 51 & ERF13 & 0,2141 & 0,6223 & 0,4820 & 3700,3 & Nodaway - NPW \\
\hline 52 & RHF2 & 0,2200 & 0,6205 & 0,4741 & 1368,0 & Rio Verde - Santos \\
\hline 53 & $\mathbf{R} 21$ & 0,9458 & 0,6096 & 0,4729 & 1870,0 & Canarana - Paranaguá \\
\hline 54 & ERF7 & 0,1886 & 0,5995 & 0,4711 & 3948,1 & Knox - NPW \\
\hline 55 & ERF2 & 0,2473 & 0,5986 & 0,4615 & 4206,0 & Champaign - NPW \\
\hline 56 & ERF3 & 0,2345 & 0,5814 & 0,4518 & 4214,1 & McLean - NPW \\
\hline 57 & R17 & 0,5134 & 0,5341 & 0,4514 & 1073,0 & Jataí - Santos \\
\hline 58 & ERF4 & 0,2360 & 0,5309 & 0,4445 & 4620,0 & Plymouth - NPW \\
\hline 59 & ERF15 & 0,1935 & 0,5087 & 0,4396 & 4554,8 & New Madrid - NPW \\
\hline 60 & ERF1 & 0,2469 & 0,4863 & 0,4346 & 4267,2 & Edgard - NPW \\
\hline 61 & ERF8 & 0,2237 & 0,4787 & 0,4301 & 4080,8 & Clinton - NPW \\
\hline 62 & RF5 & 0,4752 & 0,4661 & 0,4178 & 1462,0 & Jataí - Paranaguá \\
\hline 63 & R10 & 0,3499 & 0,4587 & 0,4143 & 963,0 & Dourados - Paranaguá \\
\hline 64 & ERF14 & 0,1747 & 0,4562 & 0,4090 & 3818,8 & Audrain - NPW \\
\hline 65 & RF7 & 0,8134 & 0,4541 & 0,4067 & 2069,0 & Canarana - Paranaguá \\
\hline 66 & R16 & 0,4998 & 0,4449 & 0,4032 & 1278,0 & Jataí - Paranaguá \\
\hline 67 & RF3 & 0,3272 & 0,4350 & 0,3966 & 1741,9 & Dourados - Santos \\
\hline 68 & R9 & 0,3326 & 0,4325 & 0,3933 & 1018,0 & Cascavel - Santos \\
\hline 69 & R11 & 0,3430 & 0,4155 & 0,3877 & 1067,0 & Dourados - Santos \\
\hline
\end{tabular}




\begin{tabular}{|c|c|c|c|c|c|c|}
\hline 70 & RF4 & 0,1940 & 0,4128 & 0,3877 & 1601,9 & Sidrolândia - Santos \\
\hline 71 & R15 & 0,2401 & 0,3894 & 0,3743 & 973,0 & Chapadão do Sul - Santos \\
\hline 72 & R24 & 0,4977 & 0,3684 & 0,3486 & 1550,0 & Primavera do Leste - Santos \\
\hline 73 & $\mathbf{R} 20$ & 0,2102 & 0,3605 & 0,3482 & 970,0 & Cristalina - Santos \\
\hline 74 & ERF9 & 0,1647 & 0,3159 & 0,3478 & 4227,2 & Montgomery - NPW \\
\hline 75 & R19 & 0,2090 & 0,3066 & 0,3326 & 1036,0 & Rio Verde - Santos \\
\hline 76 & R14 & 0,2160 & 0,3039 & 0,3255 & 1192,0 & Chapadão do Sul - Paranaguá \\
\hline 77 & RF12 & 0,3347 & 0,2796 & 0,3193 & 2222,0 & Sorriso - Santos \\
\hline 78 & RF6 & 0,1914 & 0,2248 & 0,3181 & 1452,0 & Rio Verde - Paranaguá \\
\hline 79 & RHF3 & 0,1758 & 0,1996 & 0,3172 & 1715,0 & Cristalina - Santos \\
\hline 80 & RF8 & 0,4621 & 0,1909 & 0,3156 & 1846,0 & Primavera do Leste - Paranaguá \\
\hline 81 & R23 & 0,4917 & 0,1692 & 0,3151 & 1706,0 & Primavera do Leste - Paranaguá \\
\hline 82 & R13 & 0,1947 & 0,1385 & 0,3051 & 1147,0 & Sidrolândia - Santos \\
\hline 83 & R12 & 0,1944 & 0,1099 & 0,3036 & 1154,0 & Sidrolândia - Paranaguá \\
\hline 84 & R18 & 0,1972 & 0,1005 & 0,2828 & 1264,0 & Rio Verde - Paranaguá \\
\hline 85 & RHR12 & 0,4819 & 0,0798 & 0,2533 & 1751,0 & Primavera do Leste - Santos \\
\hline 86 & RHF5 & 0,2088 & 0,0783 & 0,2442 & 1987,0 & Canarana - Santos \\
\hline 87 & RHR2 & 0,4315 & 0,0444 & 0,2373 & 1232,0 & Jataí - Santos \\
\hline 88 & R26 & 0,3379 & 0,0430 & 0,2239 & 1914,0 & Sorriso - Santos \\
\hline 89 & RHR1 & 0,4256 & 0,0286 & 0,2128 & 1294,0 & Jataí - Santos \\
\hline 90 & R25 & 0,3324 & 0,0247 & 0,1662 & 2265,0 & Sorriso - Paranaguá \\
\hline 91 & RHR11 & 0,3270 & 0,0179 & 0,1635 & 1813,0 & Primavera do Leste - Santos \\
\hline 92 & RHF4 & 0,1356 & 0,0000 & 0,1632 & 2392,0 & Sorriso - Santos \\
\hline 93 & RF9 & 0,3210 & 0,0000 & 0,1605 & 2429,0 & Sorriso - Paranaguá \\
\hline 94 & RHR10 & 0,1372 & 0,0000 & 0,1188 & 1830,0 & Canarana - Santos \\
\hline 95 & RHR4 & 0,1818 & 0,0000 & 0,1131 & 1211,0 & Rio Verde - Santos \\
\hline 96 & ERF5 & 0,2255 & 0,0000 & 0,1127 & 4800,3 & Woodbury - NPW \\
\hline 97 & RHR9 & 0,1347 & 0,0000 & 0,1065 & 1892,0 & Canarana - Santos \\
\hline 98 & RHR6 & 0,1550 & 0,0000 & 0,0918 & 1580,0 & Cristalina - Santos \\
\hline 99 & RHR3 & 0,1774 & 0,0000 & 0,0887 & 1273,0 & Rio Verde - Santos \\
\hline 100 & RHR5 & 0,1529 & 0,0000 & 0,0764 & 1642,0 & Cristalina - Santos \\
\hline 101 & RHR8 & 0,1007 & 0,0000 & 0,0593 & 2235,0 & Sorriso - Santos \\
\hline 102 & RHR7 & 0,0994 & 0,0000 & 0,0497 & 2297,0 & Sorriso - Santos \\
\hline
\end{tabular}

Quadro 5 - Resultados da eficiência das rotas, após desempate.

Foi feita uma regressão linear entre a distância da rota e os resultados de eficiência, mas uma relação fraca foi encontrada $(0,2355)$, mesmo se considerando apenas rotas com menos de $2.000 \mathrm{~km}$, a relação muito fraca se manteve $(0,1259)$. 
O Quadro 6 sumariza os resultados do Quadro 5 por quantidade de ocorrência de determinada característica comum entre todas as rotas analisadas. Por exemplo, existem rotas com um, dois ou três modais. Entre 30 rotas mais eficientes, foram encontradas $9 \mathrm{com}$ apenas um modal e 21 com dois (conforme indicado no Quadro 6). Paralelamente, entre as menos eficientes, foram encontradas também 9 com um modal, 6 com dois e 15 com três (Quadro 6). Interpretação semelhante deve ocorrer para as colunas que apontam as características: tipo de modal presente na rota (rodoviário, ferroviário e hidroviário) e país.

\begin{tabular}{lccccccccc}
\hline $\begin{array}{l}\text { Resultados } \\
\text { Consolidados }\end{array}$ & Empates & Um & Dointidade de Modais & Três & Rodoviário & Ferroviário & Hidroviário & EUA & Brasil \\
\hline $\begin{array}{l}30 \text { mais } \\
\text { eficientes }\end{array}$ & 0 & 9 & 21 & 0 & 23 & 15 & 13 & 13 & 17 \\
$\begin{array}{l}30 \text { menos } \\
\text { eficientes }\end{array}$ & 0 & 9 & 6 & 15 & 29 & 8 & 15 & 2 & 28 \\
\hline
\end{tabular}

Quadro 6 - Resultados sumarizados por características de rotas mais e menos eficientes.

Observa-se, no Quadro 6, uma preponderância de rotas norte-americanas entre as mais eficientes e brasileiras entre as menos eficientes, o que está alinhado como relatório anual norte-americano que compara o transporte de soja entre os dois países (SALIN, 2015). Entre os mais eficientes, predominam rotas com dois modais, entre os menos eficientes, rotas com três, sugerindo que existe um limite na comodalidade para que haja eficiência. Isso também foi apontado por Panagakos (2016) na sua análise do contexto europeu. O modal rodoviário é praticamente obrigatório em todas as rotas por ser um modal com a flexibilidade necessária para buscar a colheita em cada fazenda e levá-la até os pontos de transbordo para outros modais, portanto, o modal rodoviário é predominante entre todas as rotas. Em seguida, entre as eficientes predomina o modal ferroviário (bem próximo ao hidroviário). Entre as rotas ineficientes predomina, em seguida, o modal hidroviário. Marquez e Cantillo (2013), ao analisarem corredores de exportação colombianos, concluem que o modal hidroviário é o de menor custo. Entre as limitações do presente trabalho, o fato de não ter incorporado custo (devido à indisponibilidade da informação) pode ter levado a considerar o modal hidroviário menos eficiente que o ferroviário, na maioria dos casos.

\section{CONSIDERAÇÕES FINAIS}

Salin (2015) menciona que as rotas partindo do Mato Grosso (MT) tendem a custar mais do que as rotas norte-americanas, o que é validado pelos resultados encontrados neste trabalho. As rotas brasileiras menos eficientes tendem a ser aquelas que saem de MT. Além disso, Salin afirma que algumas rotas da região Sul do Brasil, destacadamente as do Paraná (PR), tendem a custar menos do que várias rotas norte-americanas, destacadamente as que partem do Estado de Iowa para os portos do Pacífico. Isso vai ao encontro dos resultados deste trabalho, pois 
as rotas brasileiras mais eficientes são da região Sul. A rota brasileira mais eficiente é a F3, de Guarapuava (PR) a Paranaguá.

Oliveira e Cicolin (2016) também mencionam que as rotas do Paraná se mostram mais eficientes. Os mesmos autores também afirmam haver relação entre eficiência e distância. Embora predominem rotas ineficientes entre aquelas com extensão superior a $2.000 \mathrm{~km}$, o presente trabalho encontrou uma relação fraca entre extensão da rota e eficiência $(0,2355)$. Oliveira e Cicolin (2016) concluem que as rotas com intermodalidade e com o modal hidroviário fluvial tendem a ser mais eficientes. Panagakos (2016) afirma que as rotas com intermodalidade são preferidas por priorizar a sustentabilidade. Marquez e Cantillo (2013), ao analisarem custos internos e externos de rotas de exportação colombianas, também concluem que o modal hidroviário fluvial possui menor custo, seguido pelo ferroviário. Isso corrobora os resultados de Oliveira e Cicolin (2016). Este trabalho apresentou rotas com dois modais entre as mais eficientes, porém as com três modais estavam entre as menos eficientes, sugerindo que existe um limite para a intermodalidade.

O modal ferroviário, no caso brasileiro, se revelou mais eficiente que o hidroviário, enquanto, no caso norte-americano, a situação se mostrou inversa. Uma explicação possível é o fato de o presente trabalho considerar apenas as hidrovias do Paraná-Tietê e da Lagoa do Patos, cuja capacidade de carga é limitada devido a restrições estruturais (em média, um reboque empurra três barcaças com capacidade de carga máxima, em média, de 1.287 toneladas) (AHRANA, 2013) (ALIANÇA, 2016), enquanto, Oliveira e Cicolin (2016) analizaram hidrovias na região Norte, cuja capacidade de carga é possivelmente maior. Por exemplo, nos EUA, em média, uma composição de barcaças é composta por três reboques empurrando 15 barcaças cuja capacidade de carga máxima é de 1.620 toneladas (WATERWAYS COUNCIL, 2016). Ou seja, em uma viagem, as composições norte-americanas transportam aproximadamente 6,2 vezes mais carga do que as brasileiras.

Considera-se que o presente trabalho demonstrou que a ferramenta DEA pode ser usada, com sucesso, para comparar a eficiência das rotas de transporte de carga. A DEA superou os problemas de distribuição de peso encontrados por Panagakos (2016). O problema de empates, gerado pela distribuição de pesos da DEA, foi superado com uso do método de desempate do índice composto e os resultados finais foram compatíveis com a literatura (SALIN, 2015) (OLIVEIRA, CICOLIN, 2016).

Entre as limitações do presente trabalho, está o fato de não se conseguir aferir, para um contexto de análise tão amplo, fatores impactantes na eficiência, por exemplo, qualidade da pista, rapidez do atendimento dos operadores, flexibilidade e rapidez no atendimento nos postos de fiscalizações. Alguns desses fatores são subjetivos e sua mensuração é dificultada. Panagakos (2016) também enumerou este, entre os desafios encontrados. Para trabalhos futuros, recomenda-se a aplicação da mesma técnica num contexto menor, por exemplo, apenas rodovias de uma pequena região economicamente relevante, em que todos esses fatores podem ser levantados com precisão.

Para trabalhos futuros, recomenda-se expandir variáveis, como, custos de transporte, custos de manutenção de rotas, desequilíbrio comercial, prazo de entrega, tipo de carga, 
disponibilidade de silos e uma variável que represente valores sociais, como a qualidade de vida do operador. Também é recomendado explorar outras possibilidades modelos DEA, como rede e modelos dinâmicos; análise janela estrutural e de desempate com o índice triplo (MARIANO; REBELATTO, 2014).

\section{Agradecimentos}

Os autores agradecem à Fundação de Amparo a Pesquisa do Estado de São Paulo (FAPESP) por financiar este projeto.

\section{ReFERÊNCIAS}

ADLER, N.; YAZHEMSKY, E. Improving discrimination in data envelopment analysis: PCADEA or variable reduction. European Journal of Operational Research. Londres, p. 273284, jan. 2010.

AHRANA, Administração da Hidrovia do Paraná. Dados e Informações Hidrovia do Rio Paraná. São Paulo: Ahrana, 2012, 52 p. Disponível em: http://www.ahrana.gov.br/dados_ informacoes.html. Acessado em: jun. 2016.

. Estatísticas de Movimentação de Carga. São Paulo: Ahrana, 2013, 26 p. Disponível em: http://www.ahrana.gov.br/dados_operacionais.html. Acessado em: jun. 2016.

A Hidrovia do Paraná. São Paulo: Ahrana, 2005, 28 p. Disponível em: http:/ /www. cooperhidro.com.br/palestras/ruy-ahrana.pdf. Acessado em: jun. 2016.

ALIANÇA (Rio Grande do Sul). Trevisa Investimentos, Frota. Disponível em: http:/ /www. trevisa.com.br/alianca/frota.html. Acessado em: jun. 2016.

ALL - AMÉRICA LATINA LOGÍSTICA S.A. (Brasil). Malha Ferroviária. Disponível em: $<$ http://pt.rumolog.com/default_pti.asp?idioma=0\&conta=45>. Acessado em: jun. 2016.

ALMEIDA, M.; MARIANO, E. B.; REBELATTO, D. A. N. Análise envoltória de dados - evolução e possibilidades de aplicação. IX Simpósio de Administração da Produção, Logística e Operações Internacionais (SIMPOI), São Paulo, 2006.

ARNOLD, J. Best practices in Management of International Trade Corridors. Trade Logistics Group. Transport Papers TP-13. The World Bank Group, Washington. DC, 2006.

BAUMEL, P. Measuring Bulk Product Transportation Fuel Efficiency. Transportation Research Forum. Washington, 2011.

BAUMEL, P.; HURBURGH, C. R.; LEE, T. Estimates of Total Fuel Consumption in Transporting Grain from Iowa to Major Grain Countries by Alternatives Modes and Routes. Iowa Grain Quality Initiative. Iowa, 2015.

BLOOMBERG NEWS. Farming the World: China's Epic Race to Avoid a Food Crisis. Bloomberg. Nova Iorque, p. 1-5, mai. 2017. Disponível em: https://www.bloomberg.com/ graphics/2017-feeding-china/. Acesso em: 28 set. 2017. 
BUREAU OF TRANSPORTATION STATISTICS. National Transportation and Statistics. United States Department of Transportation, Washington. Disponível em: http:/ / www.rita.dot.gov/bts/sites/rita.dot.gov.bts/files/publications/national_transportation_ statistics/html/table_01_26.html_mfd. Acessado em: jun. 2016.

CAMIOTO, F. C.; MARIANO, E. B.; REBELAT'TO, D. A. N. Efficiency in Brasil's industrial sectors in terms of energy and sustainable development. Environmental Science \& Policy. Londres, 2014.

CASAVANT, K., et al. Study of Rural Transportation Issues. No 147544. Research Reports. United States Department of Agriculture. Agricultural Marketing Service. Transportation and Marketing Program, 2010.

CHARNES. A., et al. Foundations of data envelopment analysis for Pareto-Koopmans efficient empirical production functions. Journal of Econometrics. Nova Iorque, 1985, p. 91-100.

CHARNES. A.; COOPER. W. W.; RHODES. E. Measuring the efficiency of the decision making units. European Journal of Operational Research. Londres, 1978, p. 429-444.

COELLI, T. J.; RAO, D. S. P.; BATTESE, G. E. An Introduction to Efficiency and Productivity Analysis, 2. ed. Massachusetts: Springer, 1998, 341 p.

COOK, W. D.; TONE, K.; ZHU, J. Data envelopment analysis: Prior to choosing a model. Omega, v. 44 n. 1-4, 2014.

DNIT - Departamento Nacional de Infraestrutura de Transportes. Anuário Estatístico das Rodovias Federais. Brasília, República Federativa do Brasil, 2010, 687 p. Disponível em: http://www.dnit.gov.br/download/rodovias/operacoes-rodoviarias/estatisticas-deacidentes/anuario-2010.pdf. Acessado em: jun. 2016.

EMATER. Acompanhamento da Safra: Safra 2014/2015. Porto Alegre: Emater, 2014, 6 p. (Séries Históricas). Disponível em: http://www.emater.tche.br/site/servicos/informacoesagropecuarias.php\#acompanhamento-de-safra. Acessado em: jun. 2016.

EMROUZNEJAD, A.; YANG, G. A survey and analysis of the first 40 years of scholarly literature in DEA: 1978 - 2016. Socio-economic Planning Sciences. Washington, p. 1-5. In-Press 2017.

FEDERAL RAILWAY ADMINISTRATION OFFICE OF SAFETY ANALYSIS. Total Accidents/Incidents Jan - Dec (2010). Disponível em: http://safetydata.fra.dot.gov/ officeofsafety/publicsite/summary.aspx. Acessado em: jun. 2016.

FERREIRA, A. N. Estudo do Efeito de Acidentes na Hidrovia Tietê-Paraná: Aspectos Preventivos,150s. Dissertação (Mestrado) - Curso de Engenharia Naval e Oceânica. Escola Politécnica da Universidade de São Paulo. São Paulo, 2000, Cap. 4.

FERROESTE (Paraná). Secretaria de Infraestrutura e Logística. Malha ferroviária. Disponível em: $\quad$ http://www.ferroeste.pr.gov.br/modules/conteudo/conteudo.php?conteudo=47. Acessado em: jun. 2016. 
GOIÁS. Instituto Mauro Borges de Estatística e Estudos Socioeconômicos. Secretaria de Estado de Gestão e Planejamento. Estatísticas Municipais: Séries Históricas, 2015. Disponível em: < http://www.imb.go.gov.br/>. Acessado em: jun. 2016.

GOLANY, B.; ROLL, Y. An Application Procedure for DEA. Omega. Oxford, v. 17, n. 3, p. 237-250, 1989.

GOOGLE COMPANY (Estados Unidos). Google Earth. Disponível em: https://www. google.com/earth/. Acessado em: jun. 2016.

GREENHOUSE GAS PROTOCOL. World Resources Institute (WRI). Calculating CO2 Emissions from Mobile Sources. 1.3. Washington, 2016, 11 p. Disponível em: http://www. ghgprotocol.org/files/ghgp/tools/co2-mobile.pdf. Acessado em: jun. 2016.

HAIR, J. F., et al. Multivariate Data Analysis. Prentice-Hall: Englewood Cliffs. NJ, 1995.

IBGE - Instituto Brasileiro de Geografia e Estatística. Ministério do Planejamento. Divisão Regional. Disponível em: http://www.ibge.gov.br/home/geociencias/geografia/default_ div_int.shtm?c=1. Acessado em: jun. 2016.

IMEA - Instituto Matogrossense de Economia Aplicada. Boletim Semanal de Soja, 358. ed. Cuiabá: Imea, 2015, 12 p. Disponível em: http://www.imea.com.br/upload/publicacoes / arquivos/R404_2015_06_19_BSSoja.pdf. Acessado em: jun. 2016.

LETA. F. R. et al. Métodos De Melhora De Ordenação em DEA Aplicados à Avaliação Estática de Tornos Mecânicos. Investigação Operacional, v. 25, n. 2, p. 229-242, 2005.

MACROLOGÍSTICA (Brasil). CNI - Confederação Nacional da Indústria. Projeto CentroOeste Competitivo. São Paulo, 2013, 75 p. Disponível em: http://arquivos.portaldaindustria. com.br/app/conteudo_18/2015/10/22/9958/ProjetoCentro-OesteCompetitivo.pdf. Acessado em: jun. 2016.

MACROLOGÍSTICA (Brasil). CNI - Confederação Nacional da Indústria. Projeto Sul Competitivo. São Paulo, 2013, 75 p. Disponível em: http://www.macrologistica.com.br/ images/stories/palestras/Projeto-Sul-Competitivo-Relatorio-Institucional-Porto\%20Alegre28-de-Junho-2011.pdf . Acessado em: jun. 2016.

MARIANO, E. B.; REBELATTO, D. A. N. Transformation of wealth produced into quality of life: analysis of the social efficiency of nation-states with the DEA's triple index approach. Journal of the Operational Research Society. Londres, p. 1.664-1.681, 2014.

MARIANO, E. B.; SOBREIRO, V. A.; REBELATTO, D. A. N. Human development and data envelopment analysis: a structured literature review. Omega. Oxford, p. 33-49, jul. 2017.

MARQUEZ, L.; CANTILLO. V. Evaluating strategic freight transport corridors including external costs. Transportation Planning and Technology, 36:6, 529-546, 2013.

MELO, I. C. Análise de Eficiência em Rotas de Transporte de Soja nos Principais Corredores Brasileiros e Norte-Americanos utilizando Análise Envoltória de Dados 
(DEA), 2017, 136s. Dissertação (Mestrado) - Curso de Engenharia de Produção, Escola de Engenharia de São Carlos, São Carlos, 2017. Cap. 2.

NASS-National Agricultural Statistics Services. Statistics by State. Disponível em: https:// www.nass.usda.gov/Statistics_by_State/. Acessado em: jun. 2016.

NATIONAL HIGHWAY TRAFFIC SAFETY ADMINISTRATION, State Traffic Safety Info. Disponível em: http://www-nrd.nhtsa.dot.gov/departments/nrd-30/ncsa/STSI/USA WEB REPORT.HTM. Acessado em: jun. 2016.

NOAA. National Oceanic and Atmospheric Administration. Distances Between United States Ports, 12. ed. Washington: Noaa, 2012, 56 p. Disponível em: http:/ /www.nauticalcharts. noaa.gov/nsd/distances-ports/distances.pdf. Acessado em: jun. 2016.

OJIMA. A. L. R. O. Análise da Movimentação Logística e Competitividade da Soja Brasileira: Uma Aplicação de Um Modelo Espacial de Programação Quadrática, 79s. Dissertação (Mestrado) - Curso de Mestre em Engenharia Elétrica. Faculdade de Engenharia Elétrica e Computação. Universidade de Campinas. Campinas, 2004.

OLIVEIRA, A. L. R.; CICOLIN, L. O. M. Evaluating the logistics performance of Brazil's corn exports: A proposal of indicators. African Journal Of Agricultural Research. Johannesburg, p. 693-700, fev. 2016.

PALLOT, J.; NEFEDOVA, T. Geographical Differentiation in Household Plot Production in Rural Russia. Eurasian Geography And Economics. Moscou, p. 40-64, jan. 2003.

PANAGAKOS, G. Green Corridors, Basics. In: PSARAFTIS. H. N. (Org.).

Green Transportation Logistics: The Quest for Win-Win Solution. Suíça, Springer, 2016. Cap. 3, p. 81-121.

ROCHA, C. B.; PARRÉ, J. L. Estudo da Distribuição Espacial do Setor Agropecuário do Rio Grande do Sul. Análise Econômica. Porto Alegre, p. 139-160, set. 2009.

SALIN, D. Soybean Transportation Guide: Brazil, 2015. U.S. Dept. of Agriculture. Agricultural Marketing Service, 2016. Disponível em: https://www.ams.usda.gov/sites/ default/files/media/Brasil\%20Soybean\%20Transportation\%20Guide\%202014.pdf Acessado em: jun. 2016.

SEAB - Secretaria de Abastecimento. Estimativas de Safra. 2015. Disponível em: http:// www.agricultura.pr.gov.br/. Acessado em: jun. 2016.

SIGA - Associação dos Produtores de Soja de Mato Grosso do Sul. Circular Técnica: Acompanhamento da Safra 2013/2014, 56. ed. Campo Grande: Siga, 2014, 9 p. Disponível em: <http://sigaweb.aprosojams.org.br/>. Acessado em: jun. 2016.

SIMOES, A. J. C., HIDALGO, C. A. The Economic Complexity Observatory: An Analytical Tool for Understanding the Dynamics of Economic Development. Workshops at the Twenty-Fifth AAAI Conference on Artificial Intelligence, São Francisco, 2011. 
TONE. K. A slacks-based measure of efficiency in data envelopment analysis. European Journal of Operational Research. Londres, p. 498-509, 2001.

TTI - Texas Transportation Institute. A Modal Comparison of Domestic Freight Transportation Effects on the General Public: Final Report. Houston, National Waterway Foundation, 2007, 69 p. Disponível em: http://www.marad.dot.gov/wp-content/uploads/ pdf/Phase_II_Report_Final_121907.pdf. Acessado em: jun. 2016.

UNITED SOYBEAN BOARD. Farm to market: a soybean's journey from field to costumer. Informa Economics, Washington, 2012. Disponível em: http://ntl.bts.gov/ lib/32000/32800/32855/STELPRDC5084108.pdf. Acessado em: jun. 2016.

US CORPS OF ENGINEERS. U.S. ARMY. Planning Center of Expertise for Inland Navigation (PCXIN), 2016. Disponível em: http://outreach.lrh.usace.army.mil/. Acessado em: jun. 2016.

VIEIRA, N. M. Caracterização da Cadeia Produtiva da Soja em Goiás, 123s. Dissertação (Mestrado) - Curso de Engenharia de Produção. Engenharia de Produção. Universidade Federal de Santa Catarina. Florianópolis, 2002.

WATERWAYS COUNCILW. Waterways Systems. Disponível em: http://waterwayscouncil. org/waterways-system/. Acessado em: jun. 2016.

Submetido em: 2-12-2016

Aceito em: 26-10-2017 